\title{
Influence of the milling parameters on the nucleophilic substitution reaction of activated $\beta$-cyclodextrins
}

\author{
László Jicsinszky ${ }^{* 1}$, Kata Tuza ${ }^{2}$, Giancarlo Cravotto ${ }^{1}$, Andrea Porcheddu ${ }^{3}$, \\ Francesco Delogu ${ }^{4}$ and Evelina Colacino ${ }^{* 1,5}$
}

\section{Full Research Paper}

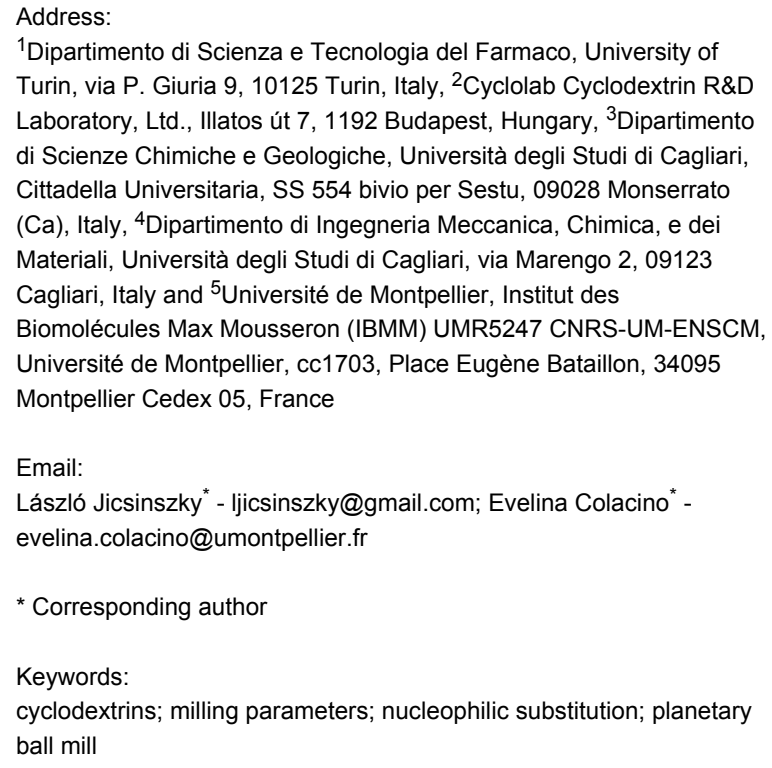

${ }^{1}$ Dipartimento di Scienza e Tecnologia del Farmaco, University of Turin, via P. Giuria 9, 10125 Turin, Italy, ${ }^{2}$ Cyclolab Cyclodextrin R\&D Laboratory, Ltd., Illatos út 7, 1192 Budapest, Hungary, ${ }^{3}$ Dipartimento di Scienze Chimiche e Geologiche, Università degli Studi di Cagliari, Cittadella Universitaria, SS 554 bivio per Sestu, 09028 Monserrato (Ca), Italy, ${ }^{4}$ Dipartimento di Ingegneria Meccanica, Chimica, e dei Materiali, Università degli Studi di Cagliari, via Marengo 2, 09123 Cagliari, Italy and ${ }^{5}$ Université de Montpellier, Institut des Biomolécules Max Mousseron (IBMM) UMR5247 CNRS-UM-ENSCM, Université de Montpellier, cc1703, Place Eugène Bataillon, 34095 Montpellier Cedex 05, France

\section{Email:}

László Jicsinszky* - ljicsinszky@gmail.com; Evelina Colacino* evelina.colacino@umontpellier.fr

* Corresponding author

\section{Keywords:}

cyclodextrins; milling parameters; nucleophilic substitution; planetary ball mill

\author{
Beilstein J. Org. Chem. 2017, 13, 1893-1899. \\ doi:10.3762/bjoc. 13.184
}

Received: 01 June 2017

Accepted: 18 August 2017

Published: 07 September 2017

This article is part of the Thematic Series "Mechanochemistry".

Guest Editor: J. G. Hernández

(C) 2017 Jicsinszky et al.; licensee Beilstein-Institut. License and terms: see end of document.

\begin{abstract}
The present work focuses on the mechanochemical preparation of industrially important $\beta$-cyclodextrin (CD) derivatives. Activated CDs have been reacted with nitrogen and sulfur nucleophiles using a planetary mill equipped with stainless steel, zirconia and glass milling tools of different sizes. It is shown that the milling frequency and the number as well as the size of the milling balls have an effect on the nucleophilic reaction.
\end{abstract}

\section{Introduction}

Their hollow structures make cyclodextrins (CDs) a class of carbohydrates that can form inclusion complexes with organic molecules, inorganic salts and complex metal ions [1]. Such a unique capacity makes $\mathrm{CD}$ derivatives crucial in a number of every-day sectors, ranging from paintings [2] to food [3]. The availability of convenient methods for their large-scale production has made CDs all but ubiquitous, including their use in a variety of investigations at the cutting edge of biological [4] and chemical science research [5]. However, there is still considerable room for the synthesis of specific CDs on the laboratory 
scale. This is the case, for instance, with $6^{\mathrm{I}}$-monoamino- $6^{\mathrm{I}}$ monodeoxy- $\beta-C D$, which is easily prepared via the reduction of the parent mono-azido derivative and is used in analytical chemistry as chiral stationary phase [6]. CDs functionalized with triazole substituents can be similarly prepared through click reactions involving the azido group as a dipolarophile [7], and utilized as suitable starting material to access hydroxy functionality after derivatization [8]. Although the preparation of carbohydrate-based complexes in a ball mill has been already reported [9-11], the use of mechanical activation for the chemical derivatization of CDs has been rather sporadic [12-15]. In this respect, it is worth noting that CDs exhibit a characteristic reactivity profile. Neither traditional synthetic routes nor a conventional carbohydrate activation methodology allow for $\mathrm{CD}$ derivatization. The major issues stem from the differing solubility of the reagents in organic solvents, meaning that high boiling polar solvents, such as DMF or DMSO, need to be used. However, these solvents are difficult to remove and usually have considerable energy contribution. Under these circumstances, the promise shown by the mechanical processing of solids of enabling chemical transformations in the absence of solvent phases renders mechanical activation extremely appealing. This is particularly true in light of the well-known capability of mechanical treatment to induce significant enhancements in chemical reactivity.

Despite the vast amount of literature on the mechanically activated synthesis of organic molecules [16-23], CD mechanochemistry offers significant challenges. For instance, the molecular weight negatively affects the reaction design and is almost one order of magnitude higher here than for common organic molecules. The laborious preparation of the starting CD-tosylate [24,25], and the considerable reactant molecular mass differences are also elements of complexity. The mechanical processing of CDs in the absence of solvent therefore promises to simplify the work-up and allows the almost complete utilization of the CD key-intermediate [13], in comparison with the classic method [6]. Moreover, the absence of a solvent, high-boiling-point ones in particular, could prevent the undesired side-reactions, that would be caused by the decomposition of DMF (formation of dimethylamine), by hydrolysis (from residual crystal water), and by alkylation and/or oxidation (DMSO) [13], leading to cleaner reaction profiles under mechanochemical conditions. Previous work on mechanically activated substitutions on tosyl ester-activated CDs resulted in high yields of the targeted 6-monoderivatized CDs, but also in complex isolation procedures due to the large number of small balls used (50 of $\varnothing 5 \mathrm{~mm}+1500$ of $\varnothing 1 \mathrm{~mm}$ steel balls) [13] Despite the longer milling times, using less balls allow outcomes to be improved [14]. This work takes the abovementioned results as a base from which to address the mechanochemical synthesis of $6^{\mathrm{I}}$-monoazido- $6^{\mathrm{I}}$-monodeoxy- $\beta$ $\mathrm{CD}$ and $6^{\mathrm{I}}-S$-monodeoxy- $6^{\mathrm{I}}$-monothiouronium- $\beta-\mathrm{CD}$ tosylate (TU- $\beta-C D$ ), an important CD intermediate for the preparation of $6^{\mathrm{I}}-S$-monodeoxy- $6^{\mathrm{I}}$-monothio- $\beta-\mathrm{CD}$ [26]. Having selected the $6^{\mathrm{I}}-O$-monotosyl- $\beta-\mathrm{CD}$ (Ts- $\left.\beta-\mathrm{CD}\right)$ as the benchmark, the nucleophilic displacement of the tosylate group in the presence of azido or thiourea (TU) nucleophiles was chosen for the study under different milling conditions. The reaction was performed in a planetary ball mill and the processing parameters were systematically varied with the aim of pointing out their influence on the nucleophilic substitution reactions in terms of rate and yield. Specifically systematic variation involved rotation speed, milling tool materials, ball number and size, ball-topowder mass ratio, the fraction of reactor volume occupied by balls and the reactor volume itself.

\section{Results and Discussion}

We previously reported [13] a successful scale-up monoazidation reaction of Ts- $\beta-C D$ (the reaction scale was $6.5 \mathrm{~g}, 5 \mathrm{mmol}$ ) in a ball-mill (Supporting Information File 1, Table S1, entries $1-4)$. Considering that the preparation of Ts- $\beta-C D$ is laborious $[24,25]$, its commercial availability is restricted by high costs and limited number of producers, the systematic investigation on the influence of the milling parameters on the reaction outcome was investigated using a reaction scale of dominantly $1 \mathrm{mmol}$ of substrate, in the presence of 3 equivalents of $\mathrm{NaN}_{3}$ or thiourea (TU) as nucleophiles (Scheme 1). Being the removal of the starting Ts- $\beta$-CD from the 6 -monoazido- $\beta-C D$ complicated due to the solubility similarities, the time to reach complete conversion ( $>99.5 \%$, defined as milling time) of the starting material had been targeted as main control parameter (see details in Supporting Information File 1).

No significant role can be ascribed to the temperature, since systematic measurements under different processing conditions indicated that it never exceeded $72{ }^{\circ} \mathrm{C}$. Further, no degradation of the activated Ts- $\beta$-CD was observed.

The yield of the mechanically induced azidation is invariably higher than the one observed in our previous work [13]. However, the rate of the reaction involving the more nucleophilic TU is considerably lower. Chemical conversion data regarding the reactions performed under different milling conditions are summarized in Table S1 (Supporting Information File 1). It can be seen that the reaction yield shows significant scatter. No definite relationship between the set of processing parameters and the yield can be identified. Nevertheless, sets of balls with different size seemingly assure the best performances in terms of yield and reaction rate, enabling full substrate conversion in shorter reaction times (Supporting Information File 1, Table S1, entries 2, 6, 11, and 12). 

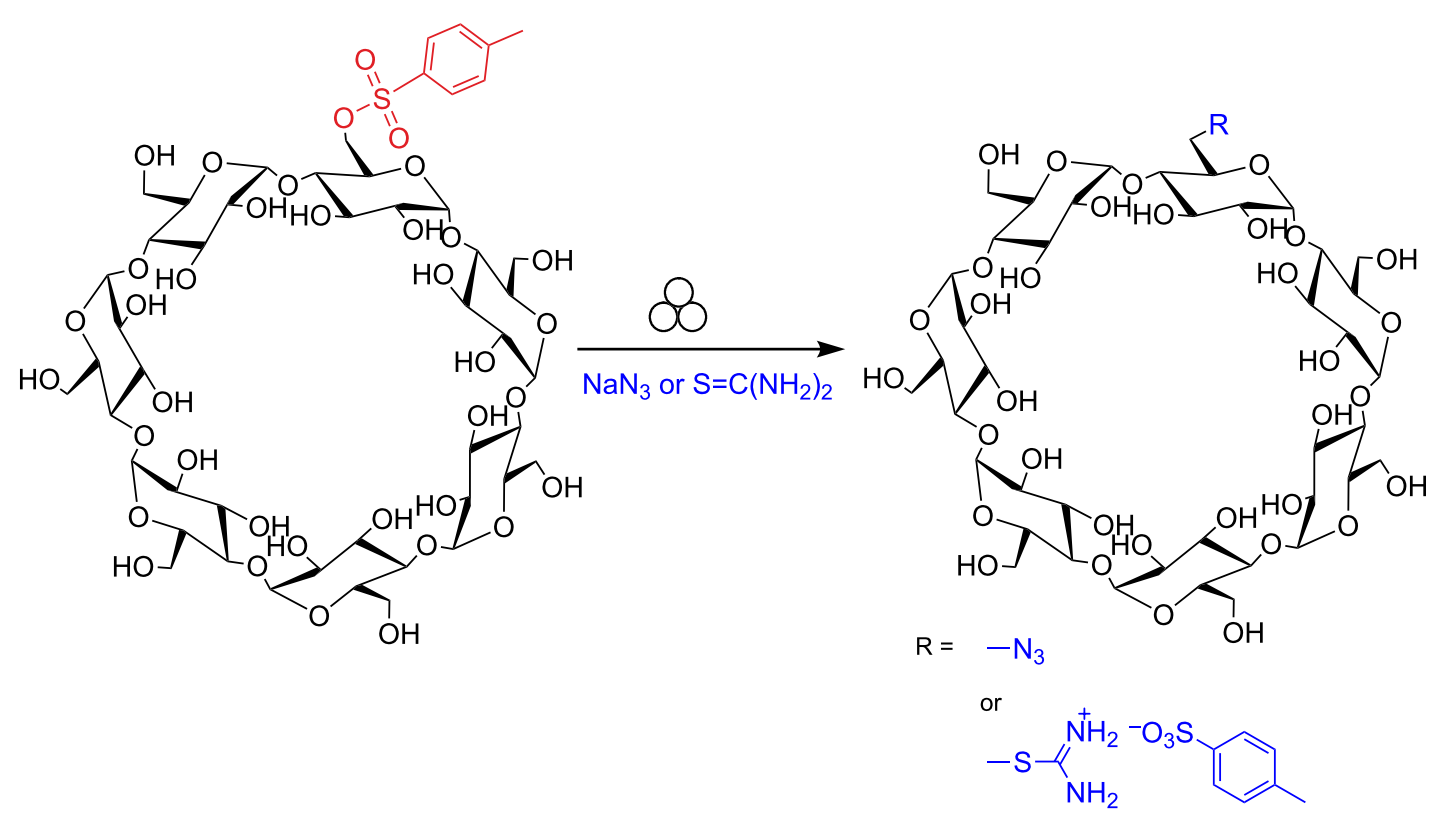

Scheme 1: Nucleophilic substitution of the 4-toluenesulfonyl group. The formalism for the mechanochemical activation was suggested by Rightmire [27].

The observed yield enhancement can be tentatively related to the effectiveness of energy transfer, which can be expected to increase as the volume occupied by balls inside the reactor increases, thus allowing milling conditions to approach frictional regimes.

In the attempt of clarifying the role of the volume fraction occupied by balls inside the reactor, the nucleophilic substitution with $\mathrm{NaN}_{3}$ was performed using glass reactors 2 and $25 \mathrm{~mL}$ in volume and the same number of balls of equal size ( 30 balls of $1 \mathrm{~mm}$ in diameter). The experimental findings are summarized in Figure 1 and Supporting Information File 1, Table S1 entries 18 and 19. The reaction rate definitely increases as the volume fraction occupied by balls inside the reactor increases. Therefore, it would appear that an increasing ball contact density shortens milling time.

Further support for the hypothesis that the higher number of impacts among balls per unit of time enhances the outcome of the reaction comes from data shown in Figure 2a and Supporting Information File 1, Table S1 entries 6 and 7. The data in Figure 2 refer to experiments performed varying the ball size while keeping the total volume occupied by balls approximately constant. Under these circumstances, the number of contacts between balls increases as the ball size decreases. Based on the above-mentioned hypothesis, reaction rate should be expected to increase. In line with expectations [18], the experimental findings indicate that the smaller the ball size, the shorter the reaction time for both nucleophiles.
Most experiments were performed at a planetary mill sun wheel speed of $550 \mathrm{~min}^{-1}$. Under these conditions, the reaction involving $\mathrm{NaN}_{3}$ as the nucleophile was investigated using the same weight of balls (ca. $45 \mathrm{~g}$ ), but varying the ratio of balls with different size. The data in Figure $2 \mathrm{a}$ and Supporting Information File 1, Table S1 entries 6-8 and 13 show no dramatic change in reaction rate. TU exhibits a slower kinetics than $\mathrm{NaN}_{3}$ under the same milling conditions (Supporting Information File 1, Table S1, entry 13 vs. 16), which hints at substratedependent reactivity (Figure $3 \mathrm{~b}$ and Supporting Information File 1, Table S1, entries 9, 11, 13 and 14 for $\mathrm{NaN}_{3}$ vs. 10, 12, 16 and 17 for $\mathrm{TU}$, respectively).

However, from the experiments the highest sun wheel speed at $650 \mathrm{~min}^{-1}$ resulted in faster reaction (Figure 2a) and the number of balls seemed to have less influence on the investigated reaction. It is assumed that a combination of the kinetic energies of the individual balls and the number of impacts can play an important role in the reaction rate.

The material constituting milling tools affects the outcomes of the substitution reaction. Data in Figure 2b (Supporting Information File 1, Table S1, entries 13 and 14 vs. 16 and 17, respectively) shows that, as far as $\mathrm{NaN}_{3}$ was used in combination with $12 \mathrm{~mm}$ balls, the best reaction yield and rate were obtained in stainless steel reactors (Supporting Information File 1, Table $\mathrm{S} 1$, entries 13 and 14). By contrast, under the same processing conditions, $\mathrm{ZrO}_{2}$ gave the best performances in reactions involving TU (Supporting Information File 1, Table S1, entries 16 


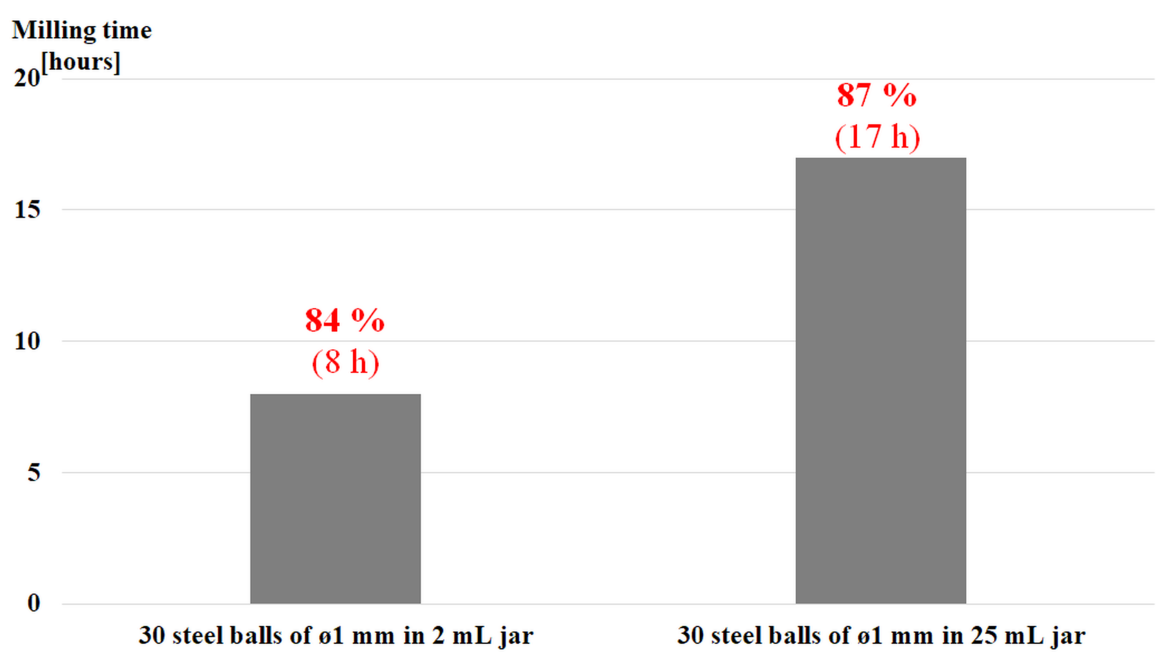

Figure 1: Effect of jar size on the reaction time using an equal number (30) of steel balls $(\varnothing 1 \mathrm{~mm})$ for the Ts $\rightarrow N_{3}$ exchange reaction in glass vials at $550 \mathrm{~min}^{-1}$ sun wheel speed.

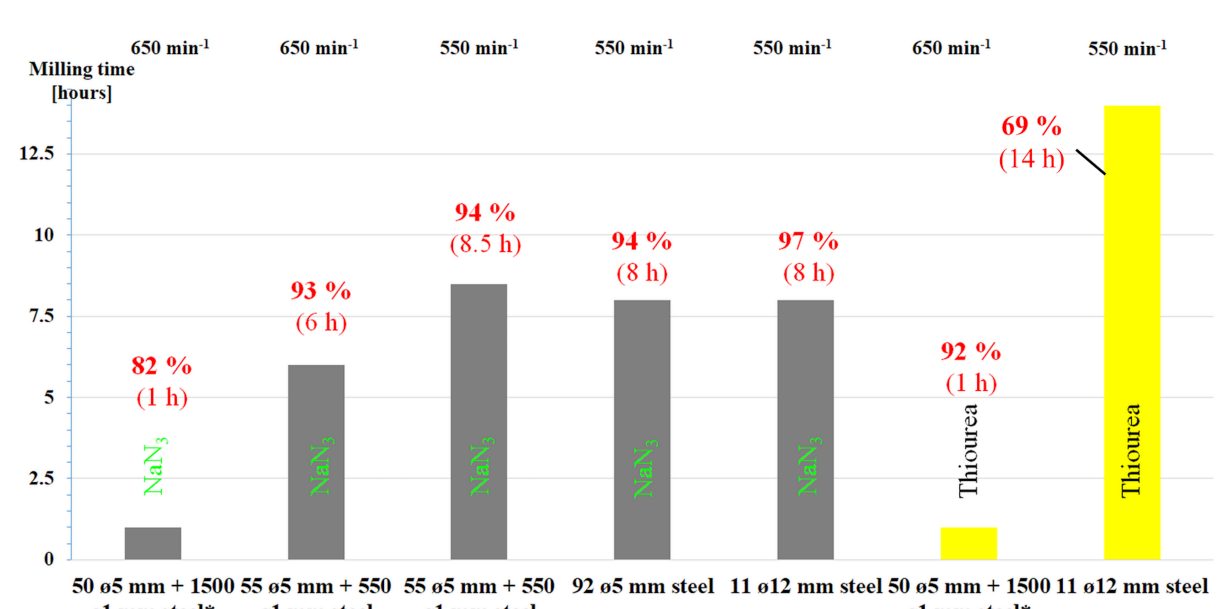

ø1 mm steel*

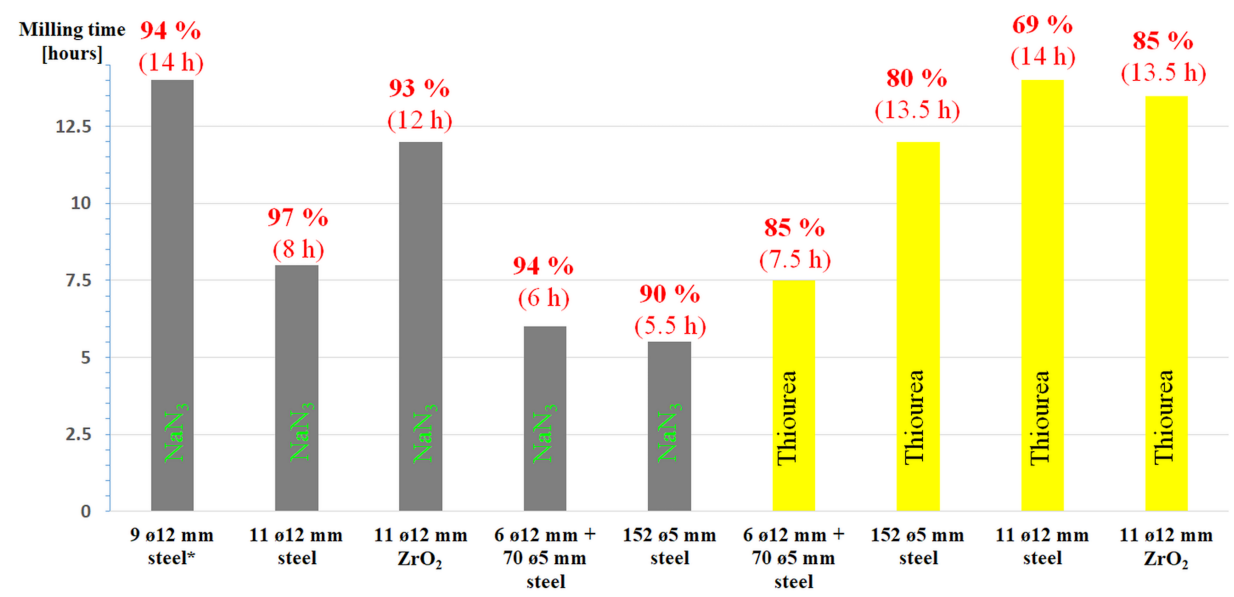

Figure 2: Effect of ball size on the reaction time to a full conversion of Ts- $\beta-C D$ : a) reactions performed at constant total steel ball weight of ca. $45 \mathrm{~g}$ (*weight of steel balls ca. $70 \mathrm{~g}$ for comparison with [13]); b) the number (and size) of balls were combined to be equal to the volume occupied by 11 balls of $\varnothing 12 \mathrm{~mm}$ (ca. $10 \mathrm{~mL}$ ) at $550 \mathrm{~min}^{-1}$ ("weight of steel balls ca. $40 \mathrm{~g}$ kept similar to 11 zirconia balls of $12 \mathrm{~mm}$ in diameter ( $\varnothing$ ) for comparison). Values given on the graph bars indicate, respectively, the yield and the reaction time to achieve full conversion of the starting Ts- $\beta-C D$. 


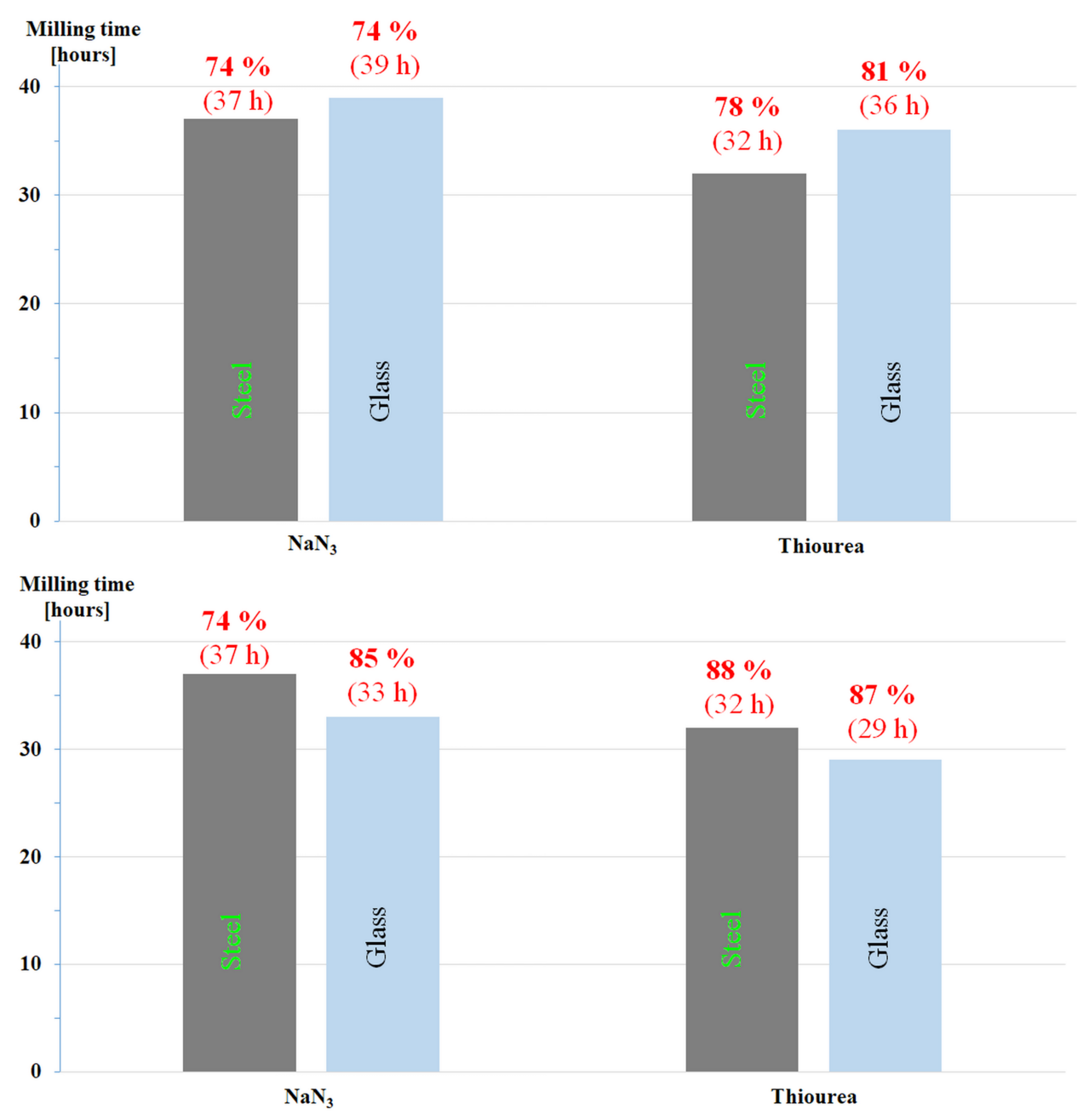

Figure 3: Reaction time as a function of ball materials at $550 \mathrm{~min}^{-1}$ in glass vials of $25 \mathrm{~mL}$ : a) equal weight: 60 steel balls of $\varnothing 1 \mathrm{~mm}(1.8 \mathrm{~g})$ vs. 20 glass balls of $\varnothing 3 \mathrm{~mm}(1.8 \mathrm{~g})$; b) 60 steel balls of $\varnothing 1 \mathrm{~mm}\left(m_{\mathrm{B}}=1.8 \mathrm{~g}, m_{\mathrm{B}} / \mathrm{m}_{\mathrm{R}}\right.$ ca. $\left.12, \Phi_{\mathrm{MB} \text {,packing }}=0.003\right) \mathrm{vs}$. $60 \mathrm{glass}$ balls of $\varnothing 3 \mathrm{~mm}$ $\left(m_{\mathrm{B}}=5.4 \mathrm{~g}, m_{\mathrm{B}} / m_{\mathrm{R}}\right.$ ca. $35, \Phi_{\mathrm{MB}}$,packing $\left.=0.077\right)$. Values given on the graphic bars indicate, respectively, the yield and the reaction time to achieve full conversion of the starting Ts- $\beta-C D$.

and 17). Thus, $\mathrm{NaN}_{3}$ seemingly displayed stronger nucleophilicity than TU when stainless steel milling tools were utilized and vice versa for $\mathrm{ZrO}_{2}$ milling tools.

In another set of experiments, for the same nucleophile, comparative experiments were performed using a total number of glass balls having the same weight $(1.8 \mathrm{~g})$ of 60 steel balls of $1 \mathrm{~mm} ø$ (Figure 3a and Supporting Information File 1, Table S1, entries 20/22 for $\mathrm{NaN}_{3}$ and 21/23 for TU).

The less hard glass balls (and jars) are in general less effective in terms of energy transfer as compared to steel. This was confirmed in the case of TU (Figure 3a and Supporting Information File 1, Table S1 entries 21 vs. 23), while milling times did not considerably change as expected [17] in the case of $\mathrm{NaN}_{3}$
(Figure 3a and Supporting Information File 1, Table S1 entry 20 vs. 22). However, an increase of the number of glass balls, led to somehow better yields after slightly shorter reaction times for both nucleophiles (Figure 3a vs. 3b), even at an improved

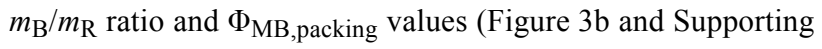
Information File 1, Table S1, entries 20, 21 vs. 24, 25).

Finally, the experimental findings collected in Supporting Information File 1, Table S1 show that for a larger volume occupied by balls inside the reactor, faster reactions were observed, independent of the material that the milling tools were made from.

\section{Conclusion}

Mechanical activation in a planetary ball mill allows the studied reactions to take place at a rate higher than the corresponding 
reactions in solution. Indeed, the nucleophilic substitution of tosyl groups is very slow at $T<80^{\circ} \mathrm{C}$ (in DMF), while in water (at $50-70^{\circ} \mathrm{C}$ ) the most competitive side reaction is the hydrolysis of the starting material. Moreover, mechanochemical activation allowed solve one of the major problems for cyclodextrin derivatization in solution. This is usually related to the very different solubilities of the reagents, thus requiring energy transfer by heating to induce reactions. Although it is difficult to reach a compromise between the reaction and side reactions, without a massive energy transfer the derivatizations are rarely successful in solution. By mechanochemistry, the reactivity is mainly affected by the sun wheel speed and the number and size of balls for both nitrogen and sulfur nucleophiles. In general, reaction rates reach a maximum as the volume fraction occupied by balls inside the reactor increases and the ball size decreases but no simple correlation was found. Consequently, it seems reasonable to connect reaction yield and rate with the total number of contact between balls. Unlike the reactivity in solution, under mechanochemical conditions the sulfur nucleophile (thiourea, TU) was less effective than the azide ion in the substitution reaction. A similar reversal of reactivity has been already observed for halogens $[12,13,28]$. The experimental findings lend support to the idea that mechanical activation can induce chemical reactivity [29] and selectivity [30] which is different to that observed in solution, which can be further complicated by the inclusion complex formation property of cyclodextrins.

How exactly the milling parameters influence the kinetics and the mechanisms of organic reactions is still question of investigation in the scientific community. Even though our contribution tries to delineate some trends, additional investigations and experiments need to be performed for a fully understanding of this still understudied and poorly understood aspect of mechanochemistry.

\section{Supporting Information}

\section{Supporting Information File 1}

Experimental procedures and technical details.

[http://www.beilstein-journals.org/bjoc/content/ supplementary/1860-5397-13-184-S1.pdf]

\section{Acknowledgements}

This work was funded by the University of Turin (Fondi Ricerca Locale 2016). The "Teach Mob - Teaching Staff Mobility Programme 2016-2017" (University of Turin, Italy) is warmly acknowledged by EC and GC. The authors thank Roquette (Lestrem, France) for the kind gift of $\beta$-cyclodextrin hydrate. AP and FD acknowledge the financial support given by the University of Cagliari (Italy).

\section{References}

1. Cyclodextrins. In Comprehensive Supramolecular Chemistry; Szejtli, J.; Osa, T., Eds.; Pergamon: New York, 1996; Vol. 3, pp 1-693.

2. Hashidzume, A.; Takashima, Y.; Yamaguchi, H.; Harada, A. Cyclodextrin. In Comprehensive Supramolecular Chemistry II; Atwood, J. L., Ed.; Elsevier: Oxford, 2017; pp 269-316. doi:10.1016/B978-0-12-409547-2.13829-6

3. Martina, K.; Binello, A.; Lawson, D.; Jicsinszky, L.; Cravotto, G. Curr. Nutr. Food Sci. 2013, 9, 167-179. doi:10.2174/1573401311309030001

4. Adeoye, O.; Cabral-Marques, H. Int. J. Pharm. 2017, in press. doi:10.1016/j.ijpharm.2017.04.050

5. Zhu, G.; Yi, Y.; Chen, J. TrAC, Trends Anal. Chem. 2016, 80, 232-241. doi:10.1016/j.trac.2016.03.022

6. Jicsinszky, L.; Iványi, R. Carbohydr. Polym. 2001, 45, 139-145. doi:10.1016/S0144-8617(00)00319-2

7. Earla, A.; Braslau, R. Macromol. Rapid Commun. 2014, 35, 666-671. doi:10.1002/marc.201300865

8. Jicsinszky, L.; Ivanyi, R. In Proceedings of the 10th International Symposium on Cyclodextrins, Ann Harbor, Michigan, USA, May 21-24, 2000; pp 46-52.

9. Rinaldi, L.; Binello, A.; Stolle, A.; Curini, M.; Cravotto, G. Steroids 2015, 98, 58-62. doi:10.1016/j.steroids.2015.02.016

10. Hedges, A.; Tenbarge, F. Cyclodextrin complexing method. U.S. Pat. Appl. 5,007,966 A, April 16, 1991.

11. Czugler, M.; Pintér, I. Carbohydr. Res. 2011, 346, 1610-1616. doi:10.1016/j.carres.2011.03.014

12. Jicsinszky, L.; Caporaso, M.; Martina, K.; Gaudino, E. C.; Cravotto, G. Beilstein J. Org. Chem. 2016, 12, 2364-2371. doi:10.3762/bjoc.12.230

13. Jicsinszky, L.; Caporaso, M.; Tuza, K.; Martina, K.; Gaudino, E. C.; Cravotto, G. ACS Sustainable Chem. Eng. 2016, 4, 919-929. doi:10.1021/acssuschemeng.5b01006

14. Jicsinszky, L.; Caporaso, M.; Gaudino, E. C.; Giovannoli, C.; Cravotto, G. Molecules 2017, 22, 485. doi:10.3390/molecules22030485

15. Menuel, S.; Doumert, B.; Saitzek, S.; Ponchel, A.; Delevoye, L.; Monflier, E.; Hapiot, F. J. Org. Chem. 2015, 80, 6259-6266. doi:10.1021/acs.joc.5b00697

16. Sánchez-Jiménez, P. E.; Valverde, J. M.; Perejón, A.; de la Calle, A.; Medina, S.; Pérez-Maqueda, L. A. Cryst. Growth Des. 2016, 16 , 7025-7036. doi:10.1021/acs.cgd.6b01228

17. Stolle, A.; Szuppa, T.; Leonhardt, S. E. S.; Ondruschka, B. Chem. Soc. Rev. 2011, 40, 2317-2329. doi:10.1039/c0cs00195c

18. Stolle, A.; Schmidt, R.; Jacob, K. Faraday Discuss. 2014, 170 , 267-286. doi:10.1039/C3FD00144J

19. Ball Milling Towards Green Synthesis: Applications, Projects, Challenges. In RSC Green Chemistry; Ranu, B.; Stolle, A., Eds.; Royal Society of Chemistry, 2015; pp 1-303. doi:10.1039/9781782621980

20. Wang, G.-W. Chem. Soc. Rev. 2013, 42, 7668-7700. doi:10.1039/c3cs35526h

21. James, S. L.; Adams, C. J.; Bolm, C.; Braga, D.; Collier, P.; Friščić, T.; Grepioni, F.; Harris, K. D. M.; Hyett, G.; Jones, W.; Krebs, A.; Mack, J.; Maini, L.; Orpen, A. G.; Parkin, I. P.; Shearouse, W. C.; Steed, J. W.; Waddell, D. C. Chem. Soc. Rev. 2012, 41, 413-447. doi:10.1039/C1CS15171A

22. Tan, D.; Loots, L.; Friščić, T. Chem. Commun. 2016, 52, 7760-7781. doi:10.1039/C6CC02015A

23. Do, J.-L.; Friščić, T. ACS Cent. Sci. 2017, 3, 13-19. doi:10.1021/acscentsci.6b00277 
24. Law, H.; Benito, J. M.; Garcia Fernandez, J. M.; Jicsinszky, L.; Crouzy, S.; Defaye, J. J. Phys. Chem. B 2011, 115, 7524-7532. doi:10.1021/jp2035345

25. Brady, B.; Lynam, N.; O'Sullivan, T.; Ahern, C.; Darcy, R. Org. Synth. 2000, 77, 220. doi:10.15227/orgsyn.077.0220

26. Liu, A.; Zhao, Q.; Guan, X. Anal. Chim. Acta 2010, 675, 106-115. doi:10.1016/j.aca.2010.07.001

27. Rightmire, N. R.; Hanusa, T. P. Dalton Trans. 2016, 45, 2352-2362. doi:10.1039/C5DT03866A

28. Konnert, L.; Dimassi, M.; Gonnet, L.; Lamaty, F.; Martinez, J.; Colacino, E. RSC Adv. 2016, 6, 36978-36986.

doi:10.1039/C6RA03222B

29. Konnert, L.; Reneaud, B.; de Figueiredo, R. M.; Campagne, J.-M.; Lamaty, F.; Martinez, J.; Colacino, E. J. Org. Chem. 2014, 79, 10132-10142. doi:10.1021/jo5017629

30. Hernández, J. G.; Bolm, C. J. Org. Chem. 2017, 82, 4007-4019. doi:10.1021/acs.joc.6b02887

\section{License and Terms}

This is an Open Access article under the terms of the Creative Commons Attribution License

(http://creativecommons.org/licenses/by/4.0), which permits unrestricted use, distribution, and reproduction in any medium, provided the original work is properly cited.

The license is subject to the Beilstein Journal of Organic Chemistry terms and conditions:

(http://www.beilstein-journals.org/bjoc)

The definitive version of this article is the electronic one which can be found at:

doi:10.3762/bjoc. 13.184 